\title{
Fenômeno de congruência e não congruência sobre a Função Exponencial no Caderno do Professor do estado de São Paulo
}

Patrícia Costa Ginez ${ }^{1}$ (i)

Secretaria de Educação do estado de São Paulo, Sorocaba, SP, Brasil

Rogério Fernando Pires ${ }^{2}$

Universidade Federal de Uberlândia, Instituto de Ciências Exatas e Naturais do Pontal, Ituiutaba, MG, Brasil

\section{Resumo}

O presente artigo é o resultado de um estudo cujo objetivo foi analisar como são propostas a mobilização e a coordenação de diferentes registros de representação semiótica da função exponencial no material de apoio do Currículo do Estado de São Paulo, o Caderno do Professor. Os pressupostos teóricos fundamentaram-se na Teoria dos Registros de Representação Semiótica de Raymond Duval, que discute a importância das variadas representações semióticas na aprendizagem matemática. As informações para a análise foram dispostas em quadros ressaltando os registros de representação de partida e os registros de representação de chegada para o estudo das transformações de acordo com a teoria de Raymond Duval. O desenvolvimento dessa pesquisa qualitativa do tipo bibliográfica apontou a mobilização, a manipulação e a coordenação de representações semióticas da função exponencial encontradas no referido material didático, que possibilitaram a compreensão do objeto matemático, por meio de distintas representações, oportunizando ao professor situações de que viabilizam a aprendizagem de seus alunos.

Palavras-chave: Função exponencial; Fenômeno de congruência e não congruência; Registro de representação semiótica.

\section{Phenomenon of congruence and non-congruence about the Exponential Function in the Teacher's notebook of the State of São Paulo}

\begin{abstract}
This article is the result of a study whose objective was to analyze how the mobilization and coordination of different records of semiotic representation of the exponential function are proposed in the supporting material of the São Paulo State Curriculum, the Teacher's Notebook. The theoretical assumptions are based on Raymond Duval's Theory of Semiotic Representation Records, which discusses the importance of various semiotic representations in mathematical learning. The information for the analysis was displayed in tables highlighting the records of representation of
\end{abstract}

\footnotetext{
${ }^{1}$ Mestre Profissional em Ensino de Ciências Exatas pela Universidade Federal de São Carlos em Educação Matemática. Professora de Matemática na rede estadual de ensino de São Paulo. E-mail: patiginez@gmail.com.

${ }^{2}$ Doutor em Educação Matemática pela Pontifícia Universidade Católica de São Paulo. Professor do Instituto de Ciências Extas e Naturais do Pontal da Universidade Federal de Uberlândia. Professor Colaborador do Programa de Pós-Graduação em Ensino de Ciências Exatas da Universidade Federal de São Carlos. E-mail: rfpires25@hotmail.com.
} 
departure and records of representation of arrival for the study of transformations according to the theory of Raymond Duval. The development of this qualitative research of the bibliographic type pointed to the mobilization, manipulation and coordination of semiotic representations of the exponential function found in the said didactic material, which enabled the understanding of the mathematical object, through different representations, providing the teacher with situations that make feasible the learning of their students.

Keywords: Exponential function; Phenomenon of congruence and non-congruence; Semiotic Representation Register.

\section{Fenómeno de congruencia y no congruencia de la función exponencial en el Cuaderno del Profesor del Estado de São Paulo}

\section{Resumen}

Este artículo es el resultado de un estudio cuyo objetivo fue analizar cómo la movilización y coordinación de diferentes registros de representación semiótica de la función exponencial se proponen en el material de apoyo del Currículo del Estado de São Paulo, el Cuaderno del Maestro. Los supuestos teóricos se basan en la Teoría de los registros de representación semiótica de Raymond Duval, que analiza la importancia de varias representaciones semióticas en el aprendizaje matemático. La información para el análisis fue desplegada en tablas destacando los registros de representación de salida y registros de representación de llegada para el estudio de transformaciones según la teoría de Raymond Duval. El desarrollo de esta investigación cualitativa de tipo bibliográfico apuntó a la movilización, manipulación y coordinación de representaciones semióticas de la función exponencial encontradas en dicho material didáctico, lo que posibilitó la comprensión del objeto matemático, a través de diferentes representaciones, dotando al docente de situaciones. que hacen factible el aprendizaje de sus alumnos.

Palabras clave: Función exponencial; Fenómeno de congruencia y no congruencia; Registro de representación semiótica.

\section{Introdução}

Muitas pessoas não se agradam da disciplina de Matemática e, por conseguinte, enxergam-na como algo difícil de ser compreendido, ocasionando dificuldades na aprendizagem. Um dos conteúdos em que recorrentemente os estudantes apresentam dificuldades é aquele que envolve a abordagem de registros algébricos, como a função exponencial, em que muitos alunos normalmente não encontram facilidade na conversão entre os diferentes tipos de representação, como língua natural, algébrica, tabelas, gráficos.

Estudos como Ginez (2020) revelam que existe uma grande quantidade de autores que buscam inovações, fazendo uso de metodologias diferenciadas, com utilização de alguns recursos com o propósito de sanar as dificuldades dos alunos referentes ao conteúdo de função exponencial.

Algumas dessas inovações concernem à utilização de recursos como tecnologias, jogos e modelagem matemática, com o intuito de explorar as funções exponenciais com as abordagens em gráficos, tabelas, expressões algébricas, na tentativa de facilitar a compreensão e a aprendizagem dos alunos. 
Tais elementos podem se constituir em materiais de apoio, por exemplo, o Caderno do Professor do Governo do Estado de São Paulo. Alguns autores discorrem a respeito, por exemplo, Cassiari (2011) comenta sobre as potencialidades e fragilidades que os materiais apresentam; Boschesi (2016) enfatiza que a utilização de tecnologias da informação e comunicação, como é proposta nesse material, muitas vezes não se apresenta com finalidade de levar o aluno a construir novos conhecimentos; e Rampini (2011) enfatiza a inibição da experiência docente e que o currículo veio para unificar, mas as atividades necessitam ser bem estudadas para identificar o que é viável ou não para a sala de aula.

Tendo em vista que esses materiais fornecem a possibilidade de ampliar a compreensão e a interpretação dos alunos e que implicitamente abordam competências e habilidades cobradas em avaliações externas, como a do Sistema de Avaliação de Rendimento Escolar do Estado de São Paulo (SARESP), somos motivados a realizar uma análise da situação de aprendizagem que compreende atividades com função exponencial.

Nesse sentido, pensamos em uma maneira de explorar os diferentes registros de representação, além de aprofundar os conceitos da Teoria dos Registros de Representações Semióticas, no tocante aos fenômenos de congruência e não congruência sobre as funções exponenciais, os quais, no material analisado, muitas vezes são apresentados em situações de aplicabilidade, com assuntos abrangendo fenômenos naturais e sociais, referentes ao crescimento populacional, meia-vida de uma substância e pela possibilidade de interdisciplinaridade da Matemática com outras áreas do conhecimento.

Nessa perspectiva, com um olhar crítico para as abordagens descritas nesse material e considerando a Matemática como apoio para as demais áreas do conhecimento, que colabora para o desenvolvimento de outras competências, realizamos uma pesquisa na tentativa de explicitar como são apresentados os registros de representação semiótica no contexto das funções exponenciais.

De acordo com os Parâmetros Curriculares Nacionais do Ensino Médio, os alunos necessitam “[...] dominar códigos e nomenclaturas da linguagem matemática, compreender e interpretar desenhos e gráficos e relacioná-los à linguagem discursiva" (BRASIL, 2007, p. 112). Ademais, salientam que, consideradas as competências e a habilidades a serem desenvolvidas referentes ao tema funções, é necessário que os alunos consigam transcrever conceitos matemáticos da linguagem natural para linguagem simbólica matemática de forma correta, o que muitas vezes é constatado como uma das dificuldades deles.

Portanto, consideramos essencial ampliar a compreensão de enunciados que se encontram em situações de aprendizagem descritas no material de apoio do professor, e o presente estudo procurou contribuir com a investigação e a análise de como são propostos os processos de construção do conceito de função exponencial. 
A pesquisa teve por objetivo analisar como são propostas a mobilização e a coordenação de diferentes registros representações semióticas de função exponencial no material de apoio ao Currículo do Estado de São Paulo, o Caderno do Professor da 1. a série do Ensino Médio, volume 2. Para tanto, o processo de análise procurou responder à pergunta norteadora: "Como o conceito de função exponencial é apresentado nesses materiais didáticos pela ótica da Teoria dos Registros de Representação Semiótica no que tange aos fenômenos de congruência e não congruência?”.

\section{Revisão de literatura}

Há uma diversidade de transformações de representação essenciais à atividade cognitiva do pensamento. Com a representação podemos reproduzir, identificar um símbolo, uma escrita, uma notação, uma imagem referente a um objeto. Entretanto, essas diferentes transformações nos registros de representação fazem com que surjam as dificuldades dos alunos, pois muitas vezes estão relacionadas com significados e funcionamento do pensamento matemático, fazendo com que confundam a representação e o objeto representado. Logo, faz-se necessária uma comunicação que leve o aluno a organizar suas ideias para a criação de novos conhecimentos que auxiliarão no entendimento e na percepção.

O pesquisador Raymond Duval desenvolveu a Teoria dos Registros de Representação Semiótica que muito contribuiu para a Educação Matemática. Duval (2011b, p. 15) enfatiza que "A análise do conhecimento não deve considerar apenas a natureza dos objetos estudados, mas igualmente a forma como os objetos são apresentados ou como podemos ter acesso a eles por nós mesmos".

Os diversos registros de representação não têm as mesmas características de um objeto, o que leva o aluno a ter dificuldade nas transformações de registros. Segundo Duval (2011b, p. 47), “A dificuldade cognitiva vem do fato que duas representações diferentes não apresentam ou não explicitam a mesma coisa do objeto que elas representam”. Para que não se confunda uma representação com o objeto representado, faz-se necessária a mobilização de diferentes registros de um mesmo objeto.

Assim, para que um sistema semiótico seja um registro de representação, precisa permitir três atividades cognitivas ligadas à semiose. De acordo com Duval (2009), a primeira delas seria a identificação do objeto matemático, ou seja, o reconhecimento de uma representação. As outras duas atividades cognitivas são os tipos de transformações de uma representação: o tratamento e a conversão. Os tratamentos são transformações no mesmo sistema de registros, isto é, transformações internas de um registro, por exemplo, iniciar com um registro numérico e realizar a operação no 
mesmo registro numérico. As conversões são transformações de uma representação em outro registro de representação, como uma representação algébrica mudar para um registro de representação gráfica.

Figura 1: Exemplo de tratamento e conversão

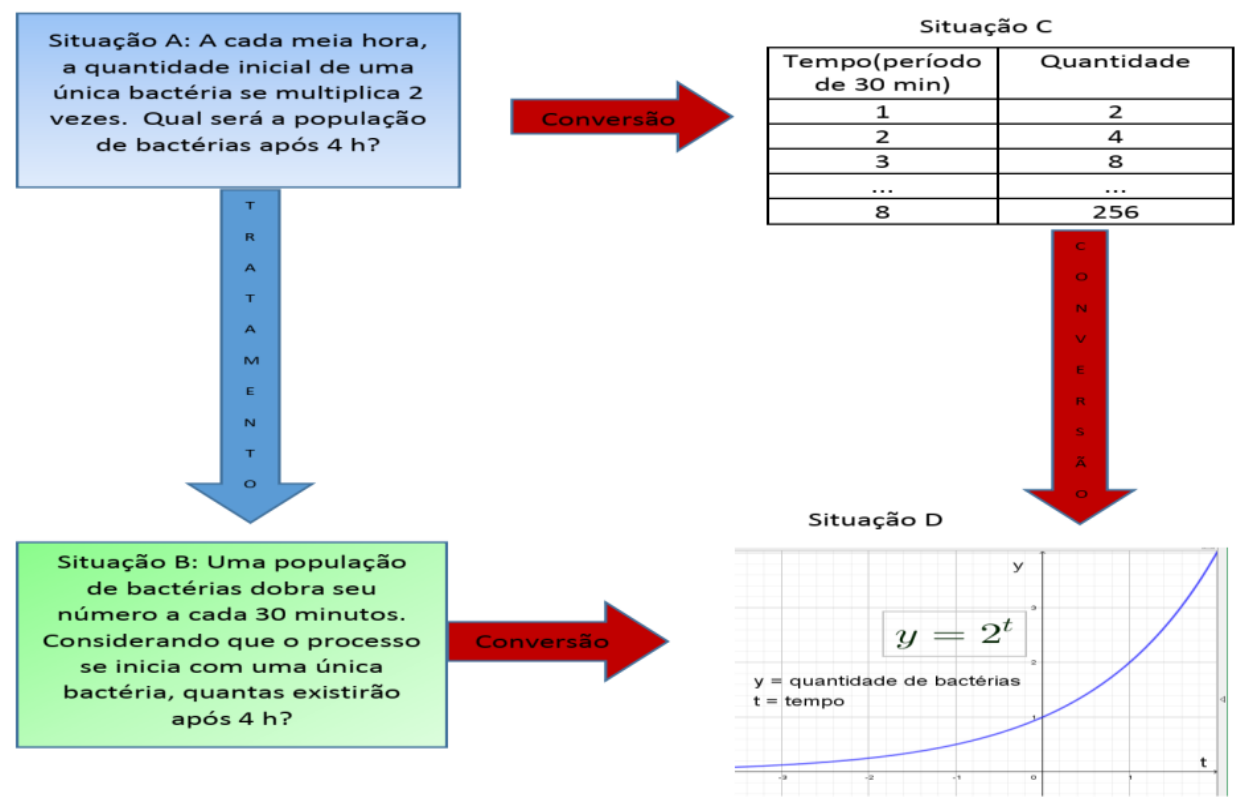

Fonte: Arquivo dos autores

A Figura 1 apresenta conteúdos diferentes, como podemos observar os registros de representações nas situações A e B, que continuam no mesmo registro, que é o registro da língua materna. Da situação A para a situação B houve uma mudança dentro do mesmo registro, ou seja, uma transformação interna de um registro, um tratamento, por meio das transformações em diferentes representações semióticas. Na situação $\mathrm{C}$, em comparação com a situação $\mathrm{A}$, transforma o registro de representação da língua materna para o registro de representação em tabelas, ocorrendo, assim, uma conversão. Na situação B para a situação D, verifica-se a transformação do registro de representação em língua materna para o registro de representação de expressão algébrica com a construção do gráfico, outra transformação em que se dá uma conversão.

É importante que o aluno consiga realizar as diferentes transformações entre os registros de representação, pois estas desenvolvem habilidades e conhecimento sobre um determinado objeto, ampliando sua compreensão matemática.

As transformações de tratamento e conversão são relevantes, porém as conversões podem ocasionar dificuldades na compreensão matemática, uma vez que a forma de representar o objeto de estudo muda, porém o objeto representado permanece o mesmo.

Um registro de representação na língua materna, os enunciados, pode causar a incompreensão dos alunos pelo fato de apresentar um linguajar diferente do que eles estão acostumados. A interpretação e a forma como coletar esses dados para uma transformação em um outro registro de 
representação necessitam ser trabalhadas para que os estudantes transponham o que Duval (2011a) chama de distanciamento da língua materna para as demais representações utilizadas na Matemática.

Os registros de representações de gráficos, por sua vez, muitas vezes favorecem a compreensão pela facilidade de visualização e pela forma simples em que se apresentam, e a visualização é um dos aspectos defendidos por Duval (2011b) para auxiliar a compreensão, quando se trata de uma abordagem global das propriedades figurais da representação gráfica de uma função.

Sobre as representações gráficas, Duval (2011a) aponta três regras semióticas de correspondência. A primeira é a abordagem ponto a ponto, que, por meio de um par de números, permite a identificação de um ponto que, mediante a localização em um plano com dois eixos, possibilita traçar um gráfico correspondente a uma expressão algébrica. A segunda é a extensão do traçado que leva em conta os dados que este fornece. A terceira é a interpretação global que faz uma análise da imagem formada com os traçados e as expressões algébricas, que propicia uma associação entre as unidades simbólicas significativas do registro algébrico com as variáveis visuais pertinentes do registro gráfico.

Os alunos realizam com tranquilidade as transformações do registro de representação de uma expressão algébrica para o registro de representação de um gráfico, utilizando ponto a ponto. No entanto, quando se deparam com o inverso, surgem as dificuldades, pois exige-se uma interpretação global da imagem fornecida pelo gráfico até chegar à expressão algébrica que representa.

A interpretação das representações gráficas cartesianas depende de uma identificação precisa de todos os valores das variáveis visuais pertinentes e do reconhecimento qualitativo das unidades da expressão simbólica correspondente (DUVAL, 2011a, p. 111 - grifo do autor).

Para a identificação precisa, os alunos necessitam saber as propriedades das representações gráficas para realizar uma interpretação global, bem como conhecer as propriedades das expressões algébricas para efetuar as identificações necessárias para a transformação dos registros de representação. Quando o aluno que se deparar com essa situação e não apresentar dificuldade, significa que ele consegue transitar pelos diferentes registros a ponto de identificar as propriedades de cada um para realizar a transformação do tipo conversão. Caso contrário, o aluno encontrará obstáculos por não distinguir as diferenças nos conteúdos.

Nesse sentido, as conversões apresentam dois fenômenos: de congruência e não congruência. Para determinar se a conversão entre dois registros de representações é congruente ou não, classificamos em unidades significantes, a fim de que possam ser colocadas em correspondência. Separamos então em registro de representação de partida que comparamos com um registro de representação de chegada. Consideramos, por exemplo, uma construção de gráfico a partir de uma expressão algébrica, na qual será considerado o registro de representação de partida, enquanto na 
construção do gráfico será observado o registro de representação de chegada. Para a identificação da conversão, faz-se necessária a comparação entre os termos significantes, e, caso o registro de representação de chegada deixar transparecer o registro de representação de partida, teremos uma conversão congruente. Contudo, se o registro de representação de chegada não transparecer de forma espontânea o registro de partida, consideraremos que a conversão é não congruente.

Quando há congruência entre a representação de partida e a representação de chegada, a conversão é trivial e poderia quase ser considerada, intuitivamente, como um simples código. Quando não há congruência, não somente a conversão torna-se custosa em termos de tempo de tratamento, mas pode criar um problema diante do qual o sujeito se sente desarmado e a possibilidade de conversão não vem mais à mente (DUVAL, 2012a, p. 283-284).

Assim, uma conversão não congruente traz dificuldades ao aluno pelo fato de ser considerada menos espontânea e mais difícil de ser compreendida, tendo em vista que a conversão não vem à mente e o aluno não consegue acessar conhecimentos em sua memória, de modo que reconheça as correspondências das unidades significantes para a realização da conversão.

O reconhecimento das correspondências e não correspondências de um objeto em relação ao registro de partida e ao registro de representação de chegada acontece com a variação do conteúdo para efetuar uma conversão diferente da realizada anteriormente, o qual é um dos motivos da incompreensão dos alunos.

Para estabelecer uma congruência, embora não sejam suficientes, necessitamos de três critérios como descrito por Duval (2009).

I - Uma correspondência semântica entre suas unidades significantes.

II - Univocidade semântica terminal: a cada unidade significante de registro de representação partida corresponde uma só unidade significante de registro de representação de chegada.

III - Existência de correspondência de mesma ordem de apreensão das unidades significantes em cada uma das duas representações.

Se o registro de representação atender aos três critérios, teremos um fenômeno de congruência; caso considere somente a alguns dos critérios ou nenhum, dizemos que ocorre o fenômeno de não congruência.

A compreensão matemática, segundo a Teoria dos Registros de Representação de Duval, pode ser alcançada por meio da habilidade do aluno em transitar nos diferentes registros de representação. Nesse sentido, realizamos uma análise de questões apresentadas em situações de aprendizagem, que envolvem alguns registros de representação, com foco nos fenômenos de congruência e não congruência, visando a um auxílio para a aprendizagem e compreensão das atividades matemáticas. 


\section{Metodologia}

O estudo de caráter qualitativo empregando a técnica de análise bibliográfica, de acordo com Gil (2002), consiste em analisar materiais publicados, como livros, revistas, enciclopédias, artigos científicos, entre outros. A pesquisa aqui relatada foi motivada pelo fato de os alunos apresentarem dificuldades na execução de atividades envolvendo as noções de função exponencial, que são propostas em materiais que se constituíram como fonte de dados para a realização deste estudo, como o Caderno do Professor.

No trabalho, analisamos o Caderno do Professor utilizado na rede estadual de ensino de São Paulo, que contempla a 1. ${ }^{a}$ série do Ensino Médio sobre o conteúdo função exponencial, situado no volume 2, Situação de Aprendizagem 1.

Embora 2019 tenha sido um ano de transição, em que os materiais iniciaram uma reconstituição à luz da Base Nacional Comum Curricular (BNCC), com caderno de exercícios que exploravam função exponencial, os professores da rede estadual de São Paulo continuaram contando com o mesmo caderno.

Contemplando o objetivo deste trabalho de verificar como o conceito de função exponencial é apresentado nesse material didático pela ótica da teoria dos Registros de Representação Semiótica no que tange aos fenômenos de congruência e não congruência, decidimos adotar três categorias de análise: tratamento, conversão congruente e não congruente.

Identificamos o que o Caderno do Professor contempla sobre os diferentes registros, e, para uma melhor organização, utilizamos quadros, com inspiração no método de Cornell, que divide as anotações em três seções, cada qual com um objetivo específico, porém a ideia principal é sintetizar o material de estudo. Assim, os quadros apresentarão o enunciado da atividade, a possível solução e, por fim, a categorização da transformação de representação exigida.

A categorização apresentou a questão, sua solução e a categoria de análise a que ela pertence. Portanto, ao identificar um tratamento, uma das categorias de análise, sinalizamos em nosso quadro pela cor azul. As conversões congruente e não congruente, quando identificadas, sinalizamos com um contorno laranja, e, caso ela seja congruente, seu contorno será verde; se não for congruente, seu preenchimento será vermelho.

As questões analisadas compreenderam pelo menos uma das categorias de análise ou até mesmo as três, pois consideramos importantes atividades que abordam mais de um registro de representação e que não apresentam somente o tratamento, mas as conversões, que são os pontos em que os alunos encontram mais dificuldades. 


\section{Resultados}

Nossos estudos tiveram como foco a análise de atividades selecionadas, identificando caminhos para realizar o tratamento e a conversão dos registros de representação à luz da Teoria dos Registros de Representação Semiótica de Duval, com intuito de observar como os fenômenos de congruência e não congruência se apresentam no Caderno do Professor. Entre as atividades apresentadas no Caderno do Professor, considerando as questões que se assemelhavam em abordagens e registros de representação, optamos por inserir algumas delas nos resultados, e a análise de todas questões se encontram em Ginez (2020).

Na primeira atividade, após uma seção de leitura e análise de texto que exploram conceitos de potência, seria necessário que os alunos solucionassem a questão apresentada no Quadro 1. No registro de partida colocamos a questão e no registro de chegada, a resolução da questão e, $\operatorname{logo}$ abaixo, destacamos a categorização, identificando um tratamento, uma conversão congruente ou uma conversão não congruente.

Para essa identificação, são necessárias as comparações da questão com a resolução, ou melhor, a comparação do registro de representação de partida com o registro de representação de chegada.

Quadro 1: Atividade 1 - Caderno do Professor

\begin{tabular}{|c|c|}
\hline 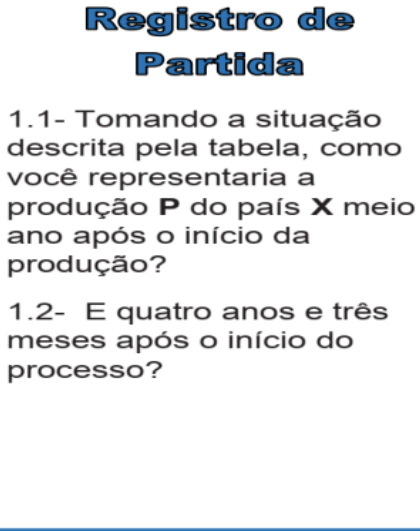 & 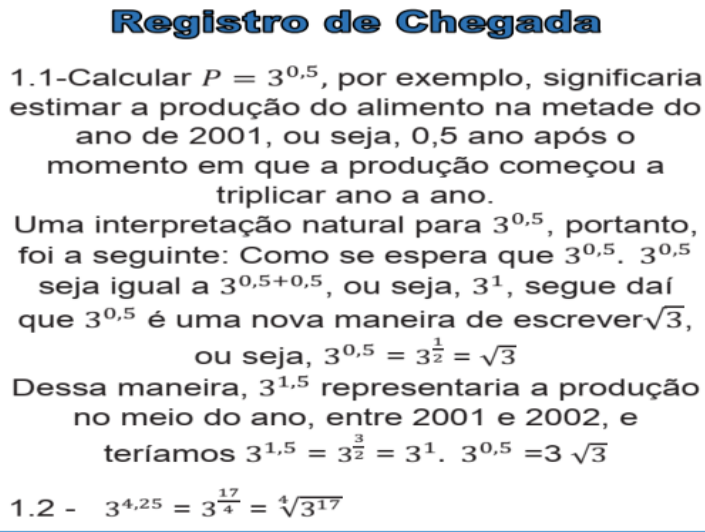 \\
\hline
\end{tabular}

\section{Conversão não congruente}

Fonte: Dados da pesquisa

No Quadro 1, ao comparar o registro de partida, que se encontra na língua materna, com o registro de chegada, que se apresenta com um registro de representação numérico, observamos que houve uma conversão. Para identificar o tipo de conversão, necessitamos analisar os dados descritos e a relação de correspondência. Considerando que $3^{0}$, tempo inicial, $3^{1}$ após um ano, então meio ano após o início do processo seria $3^{0,5}$, mas o registro de chegada final apresenta $\sqrt{3}$, o que não corresponde de maneira direta à questão meio ano após o processo. Logo, o registro de chegada não deixa transparecer de forma espontânea o registro de partida. Além disso, temos os três critérios de 
congruência descritos por Duval (2009): correspondência semântica, univocidade semântica terminal e existência de correspondência de mesma ordem. Como nesse caso não atende aos critérios, significa que existe uma conversão não congruente, como representado no Quadro 1, no qual pode-se observar que a identificação da conversão tem seu contorno laranja, e, como é uma conversão não congruente, seu preenchimento é vermelho.

O Quadro 2 apresenta uma atividade com a proposta de preencher os espaços a partir da expressão algébrica dada, substituindo a incógnita $x$ pelo número correspondente à linha, no qual destacamos as possíveis respostas em vermelho.

Quadro 2: Atividade 2 - Caderno do Professor

\begin{tabular}{|c|c|c|c|c|c|}
\hline \multirow{8}{*}{ 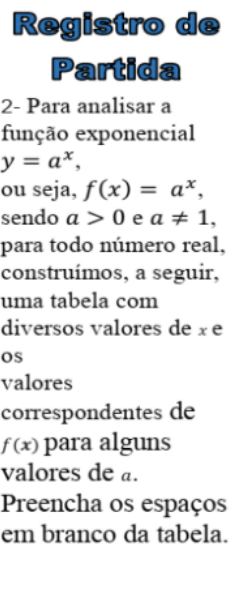 } & \multicolumn{5}{|c|}{ 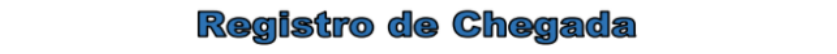 } \\
\hline & $\mathbf{x}$ & $2^{x}$ & $3^{x}$ & $\left(\frac{1}{2}\right)^{x}$ & $\left(\frac{1}{3}\right)^{x}$ \\
\hline & 1 & 2 & 3 & $\frac{1}{2}$ & $\frac{1}{3}$ \\
\hline & 2 & $2^{2}=4$ & $3^{2}=9$ & $\left(\frac{1}{2}\right)^{2}=\frac{1}{4}$ & $\left(\frac{1}{3}\right)^{2}=\frac{1}{9}$ \\
\hline & 3 & $2^{3}=8$ & $3^{3}=27$ & $\left(\frac{1}{2}\right)^{3}=\frac{1}{8}$ & $\left(\frac{1}{3}\right)^{3}=\frac{1}{27}$ \\
\hline & $\mathbf{0}$ & $2^{0}=1$ & $3^{0}=1$ & $\left(\frac{1}{2}\right)^{0}=1$ & $\left(\frac{1}{3}\right)^{0}=1$ \\
\hline & -3 & $\begin{array}{l}2^{-3}=\frac{1}{2^{3}} \\
=\frac{1}{8}\end{array}$ & $\begin{array}{l}3^{-3}=\frac{1}{3^{3}} \\
=\frac{1}{27}\end{array}$ & $\left(\frac{1}{2}\right)^{-3}=2^{3}=8$ & $\left(\frac{1}{3}\right)^{-3}=3^{3}=27$ \\
\hline & $\frac{1}{2}$ & $\begin{array}{l}2^{\frac{1}{2}}=\sqrt{2} \\
\cong 1,41\end{array}$ & $\begin{array}{l}3^{\frac{1}{3}}=\sqrt{3} \\
\cong 1,73\end{array}$ & $\begin{array}{l}\left(\frac{1}{2}\right)^{\frac{1}{2}}=\sqrt{\frac{1}{2}}=\frac{1}{\sqrt{2}} \cong \\
0,71\end{array}$ & $\begin{array}{l}\left(\frac{1}{3}\right)^{\frac{1}{3}}=\sqrt{\frac{1}{3}}=\frac{1}{\sqrt{3}} \\
\simeq 0.58\end{array}$ \\
\hline
\end{tabular}

Fonte: Dados da pesquisa

Temos que o registro de partida nos mostra uma tabela para preencher seus espaços e supõese que o aluno completará os dados, por meio de uma resolução de potências com registro numérico, após uma revisão das propriedades de potência. Podemos observar que não há mudança de registros e, segundo Duval (2009), a transformação é interna, ou seja, um tratamento, no qual identificamos no quadro com a cor azul.

O Quadro 3 nos mostra a atividade 4 do Caderno Professor, que se assemelha à atividade 3 que consta no Caderno do Professor e na análise do material de Ginez (2020). A atividade faz uma abordagem de registro algébrico para um registro de representação de gráfico. 
Quadro 3: Atividade 4 - Caderno do Professor

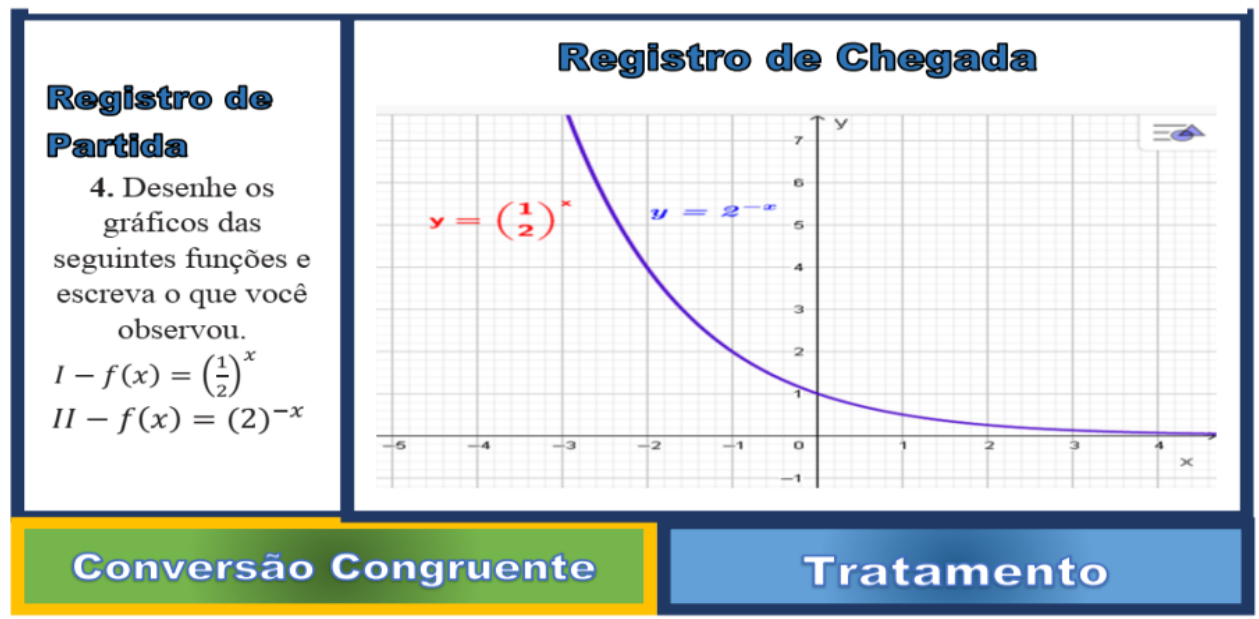

Fonte: Dados da pesquisa

Em uma representação gráfica como a indicada no Quadro 3, o aluno tem a possibilidade de fazer uma correspondência com os valores de x e y, em que poderão utilizar a relação ponto a ponto, descritas por Duval (2011a), que, mediante a localização de pontos, permite realizar o traçado do gráfico. A transformação do registro de partida - as expressões algébricas - para o registro de chegada - o gráfico - possui uma correspondência de termo a termo, a expressão algébrica representa um gráfico, ou seja, tem uma univocidade terminal e a ordem se mantém, satisfazendo os critérios de congruência estabelecidos por Duval (2009). Portanto, temos uma conversão congruente, caso seja feita uma análise global das propriedades figurais como definido por Duval (2011a).

Se analisarmos o item I e sua expressão algébrica e o item II com sua expressão algébrica, podemos comparar esses itens e explicar aos alunos que, ao olharem o gráfico, observarão apenas um traçado, o que significa que as expressões representam um mesmo objeto. Então, o que ocorre entre os itens I e II é uma transformação interna, ou seja, um tratamento, pois as duas expressões estão relacionadas a um único gráfico. A atividade mostra que o mesmo objeto matemático pode ser representado por diferentes representações.

O Quadro 4 retrata uma atividade que aborda a construção de gráficos, conforme exposto no Quadro 3, mas apresentando vários gráficos de diferentes expressões algébricas construídos em um mesmo plano. Cada expressão tem seu respectivo gráfico, que compreende os critérios de congruência defendidos por Duval (2009). Existe uma correspondência semântica termo a termo entre as unidades significantes e cada expressão tem um único gráfico, atendendo à univocidade terminal. No tocante ao registro algébrico e ao registro gráfico, a ordem se mantém. Para essa atividade, o Caderno do Professor propõe a construção com o uso de software, o que viabiliza o estabelecimento de relações entre as unidades simbólicas significativas da representação algébrica com as variáveis visuais pertinentes da representação gráfica, conforme salienta Duval (2011a). 
Quadro 4: Atividade 5 - Caderno do Professor

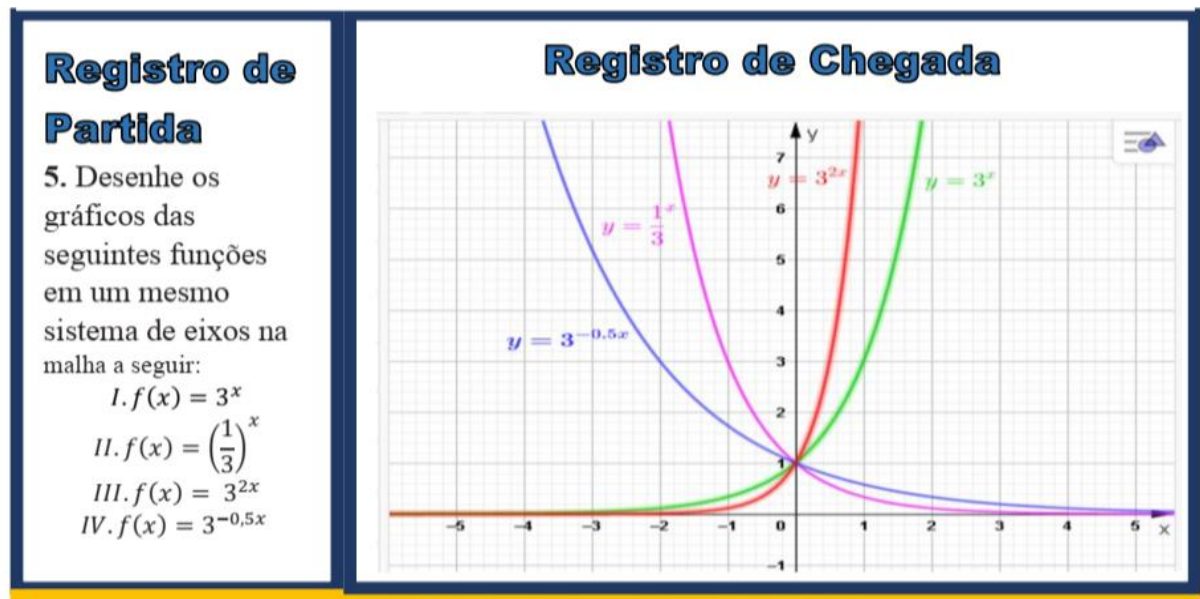

\section{Conversão Congruente}

Fonte: Dados da pesquisa

As construções gráficas como as realizadas no Quadro 4 proporcionam um tempo maior para análise, caso seja utilizado o software para a construção. Assim, elas serão executadas com rapidez e precisão, proporcionando uma facilidade na visualização, o que contribui para a compreensão matemática, por exemplo, a análise de uma expressão com o gráfico, referente a uma função crescente ou decrescente.

O Quadro 5 aborda uma continuação da atividade 5, a qual, por meio das propriedades das potências, requer que os alunos identifiquem o coeficiente $\mathrm{k}$ nas representações algébricas, verificando-se, por meio dos coeficientes, uma função crescente ou decrescente.

Com as representações algébricas apontadas para identificar o valor do coeficiente $\mathrm{k}$, faz-se necessária uma transformação do registro, o qual passa a ser escrito com um registro numérico, que por meio das propriedades das potências torna possível determinar o valor de k. Nessa atividade, ocorrem duas transformações, e uma delas é a conversão congruente da expressão algébrica para o registro numérico. A conversão é congruente, pois acontece de forma espontânea e atende aos três critérios de congruência descritos por Duval (2009). Para a determinação do valor de k, a transformação no registro numérico recorre a uma transformação interna, resultando em um tratamento. 
Quadro 5: Atividade 5 - a e b - Caderno do Professor

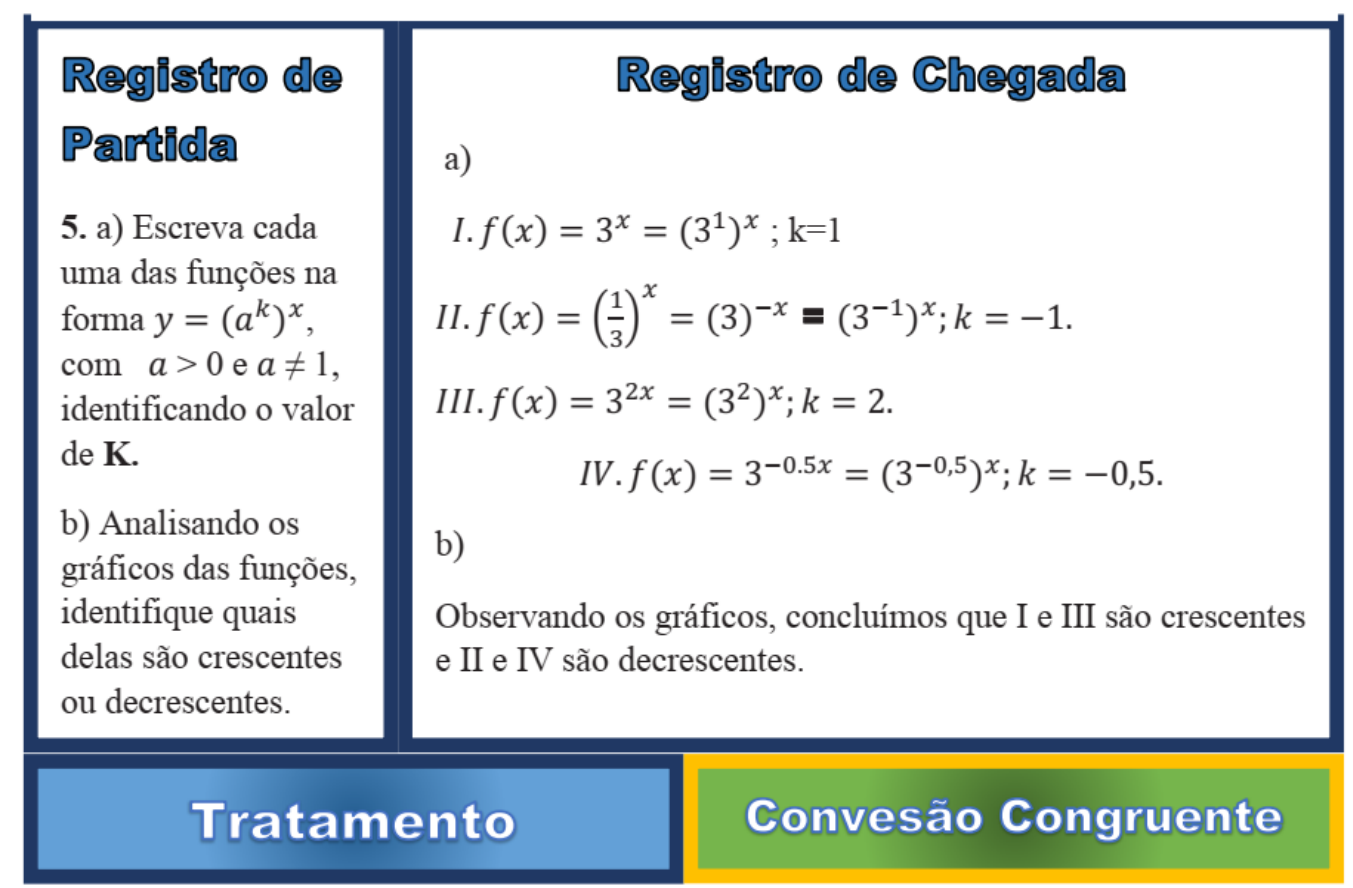

Fonte: Dados da pesquisa

No item b, atividade 5, pede-se para analisar os gráficos e determinar quais representam funções crescentes ou decrescentes. Nesse ponto, os alunos podem ser orientados a dar a resposta olhando o valor de k, mas, como se solicita examinar o gráfico, é interessante que mostre as comparações tanto dos gráficos como das expressões algébricas.

Ainda na atividade 5, representada nos Quadros 4 e 5, exploram-se duas categorias de análise: tratamento e conversão congruente.

Visando à compreensão dos alunos, é preciso que se transite pelos diferentes registros. Logo, observando-se a proposta dessa atividade, sente-se falta de uma abordagem para realizar o caminho inverso, exigindo-se uma interpretação global do gráfico representado para determinar uma expressão algébrica, que faz parte das regras semióticas de correspondência de Duval (2011a). A atividade torna-se mais complicada para o aluno, mas a interpretação de um traçado, uma curva de um gráfico para determinar a expressão algébrica correspondente, fará com que ele adquira conhecimentos sobre funções e isso contribuirá para transitar nos diferentes registros com facilidade, pois, para sanar as dificuldades nesse tipo de atividade, são essenciais abordagens que levem os estudantes a adquirir o conhecimento acerca desses diferentes registros.

$\mathrm{Na}$ atividade no Quadro 6, é necessário substituir os valores de t na expressão algébrica dada no enunciado. Esse processo inicial de transformação acreditamos que não será difícil para os alunos, pois a resolução se inicia em uma transformação de mesmo registro, ou seja, um tratamento como descrito por Duval (2009). Entretanto, na resolução dos demais itens, devem-se buscar na memória as propriedades das potências, e, nesse caso, se a conversão não vem à mente, os alunos encontram 
obstáculos, uma vez que a transformação não se apresenta de forma espontânea nem faz a relação de correspondência, tampouco atende aos critérios de congruência descritos por Duval (2009), acontecendo uma conversão não congruente.

Quadro 6: Atividade 6, a-Caderno do Professor

\begin{tabular}{|c|c|}
\hline 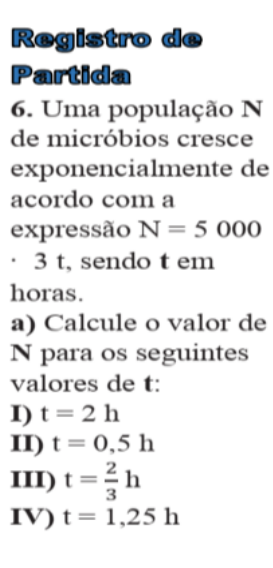 & 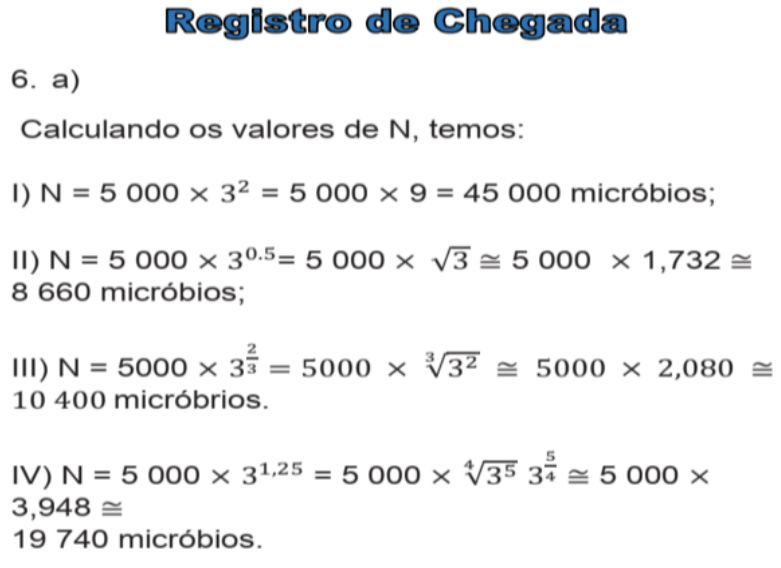 \\
\hline Tratam & Conversão não congruente \\
\hline
\end{tabular}

Fonte: Dados da pesquisa

É importante trabalhar essas atividades por apresentarem um contexto em que podemos dar exemplos e nos aproximar de situações do cotidiano, o que favorece a aprendizagem dos alunos.

O Quadro 7 apresenta uma continuidade da atividade 6, em que a proposta é uma construção de gráfico.

Quadro 7: Atividade 6, b-Caderno do Professor

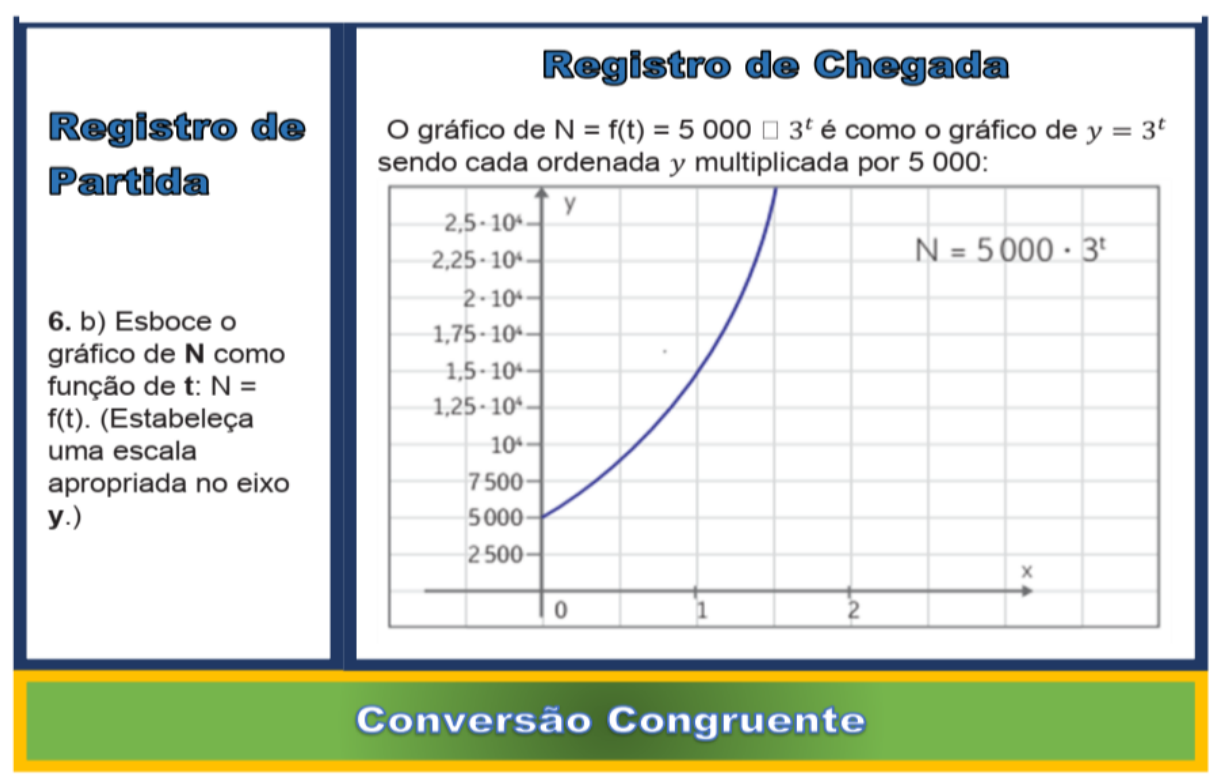

Fonte: Dados da pesquisa

A construção do gráfico por meio da expressão algébrica pode ser explorada com os dados contidos no item a, Quadro 6, em que, com tabelas, esses dados podem ser adicionados para uma 
melhor visualização e construção do gráfico. Outra observação é que o gráfico dessa função, em comparação com os demais, representa de forma diferente, indicando que houve uma multiplicação por 5 000, deixando o formato do gráfico distinto dos demais estudados até o momento.

Um fato que observamos é que o Caderno do Professor deixa de indicar situações que envolvem a multiplicação pela expressão que representa a função exponencial, não apresentando aspectos diferentes em relação aos gráficos.

Inicialmente, a atividade 7, no Quadro 8, consiste em determinar uma expressão algébrica, por meio do enunciado em língua materna, para assim estabelecer o valor no item a. A partir do registro de partida, o enunciado, ocorre uma transformação para um registro de representação algébrico, o registro de chegada, acontecendo uma conversão.

Quadro 8: Atividade 7 - Caderno do Professor

\begin{tabular}{|c|c|}
\hline 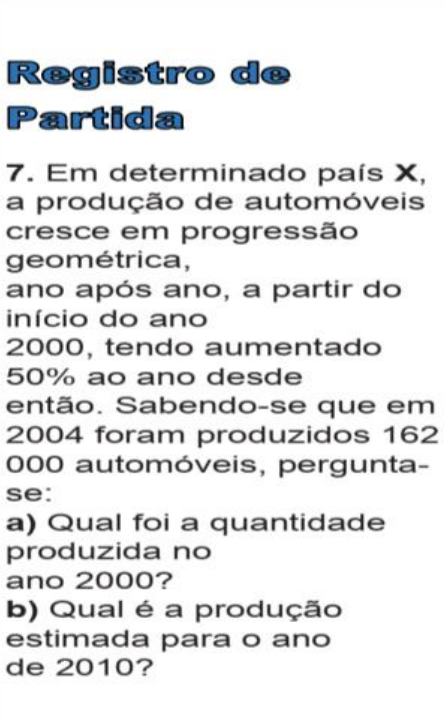 & 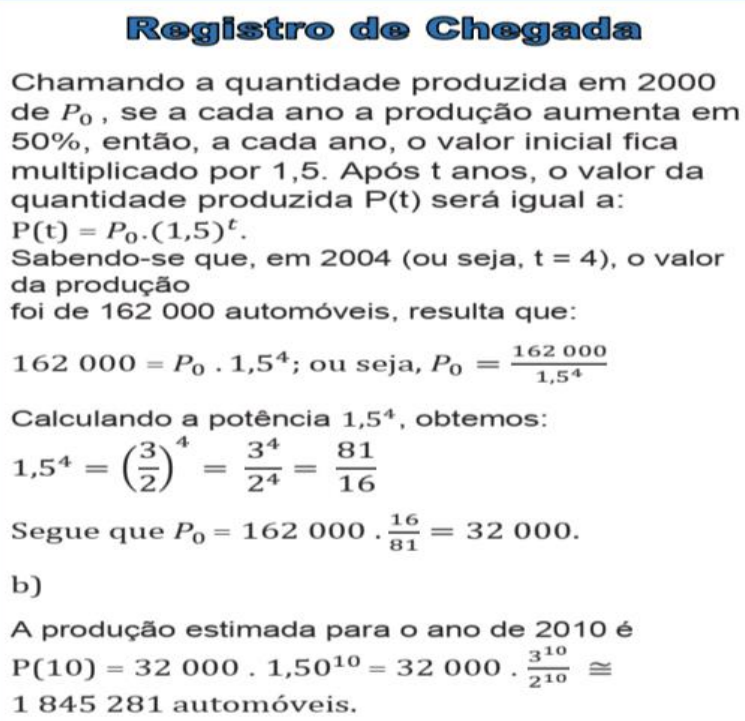 \\
\hline & Tratamento \\
\hline
\end{tabular}

Fonte: Dados da Pesquisa

Para a resolução dessa atividade, o aluno necessita de uma boa interpretação do enunciado para compreender o que se pede.

No entanto, comparando o enunciado, registro de partida, e a expressão algébrica, registro de chegada, observamos que a expressão algébrica não transparece o registro de partida, o enunciado, pois $50 \%$ de aumento ano após ano corresponde a 1,5, o que não aponta uma relação termo a termo e, por conseguinte, não atende aos critérios de congruência estabelecidos por Duval (2009), sendo considerada uma conversão não congruente. Na sequência, na resolução do item b acontece um tratamento, pois, depois da determinação da expressão algébrica no item a, substituiu o tempo por 10 e realizou uma transformação interna, dentro do mesmo registro, ou seja, um tratamento. 
Na atividade 8, Quadro 9, cada item pede a construção de um gráfico a partir de duas funções algébricas representadas em cada caso. Percebemos que na resolução dessas questões houve uma atenção voltada para os aspectos gráficos necessários para uma compreensão dos alunos referente às alterações realizadas nas expressões.

Quadro 9: Atividade 8, a - Caderno do Professor

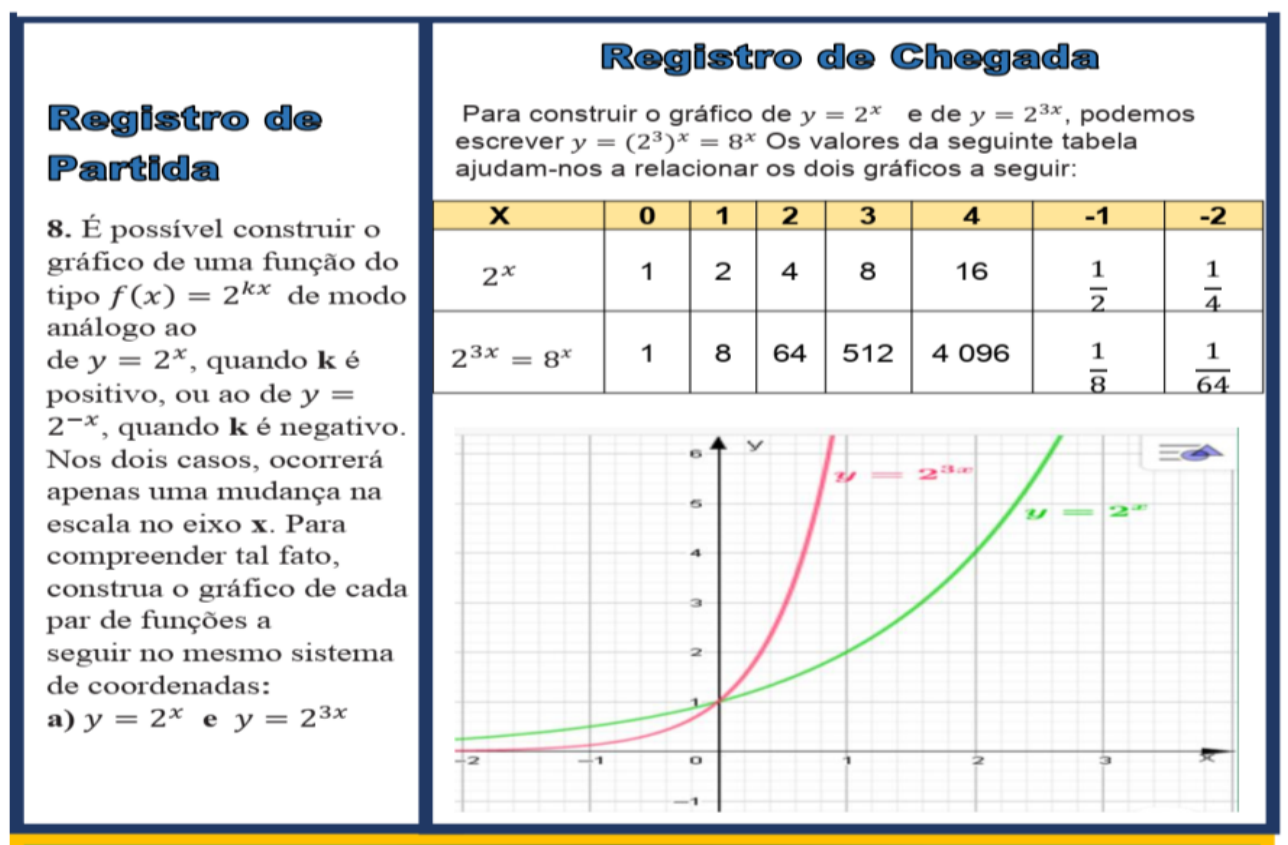

Conversão Congruente

Fonte: Dados da pesquisa

A abordagem no item a demonstra mais de uma transformação, na possível solução apresentada a partir do registro de representação algébrico. A transformação do registro algébrico para o registro em tabela ocorre de forma espontânea, por meio das substituições, a qual, para chegar ao resultado, necessita de um raciocínio lógico apurado ou utiliza uma transformação dentro do mesmo registro, isto é, um tratamento. Temos então uma conversão seguida de um tratamento. Tendo em vista a construção do gráfico a partir da tabela, observamos uma conversão congruente, pois atende aos três critérios estabelecidos por Duval (2009). Podemos verificar que a relação termo a termo é constatada por meio dos dados na tabela e que podemos localizar os pontos e realizar uma construção do gráfico fazendo uso das regras de correspondência, ponto a ponto, descritos por Duval (2011a). Com essas construções, podem ser realizadas comparações entre os parâmetros apresentados pelos gráficos e suas respectivas expressões algébricas.

$\mathrm{Na}$ continuação da atividade, deparamo-nos com construções de gráficos que podem ser realizadas conforme o exposto no Quadro 9, por meio de tabela e após uma construção do gráfico. A atividade apenas pede que se construa o gráfico. Então, a critério do professor, o nível de dificuldades 
dos alunos será um parâmetro para avaliar a necessidade de executar as atividades explorando tabelas e construções de gráficos.

A atividade 8, item b, no Quadro 10, necessita de um tratamento nas expressões algébricas seguido de uma conversão. Identificamos um tratamento pois, nas expressões algébricas, acontece uma transformação para o registro numérico seguido de um tratamento para chegar ao resultado. No entanto, esse resultado não se relaciona ao termo dado inicialmente, para o que percebemos a necessidade de buscar conhecimentos sobre as propriedades de potência.

Quadro 10: Atividade 8, b-Caderno do Professor

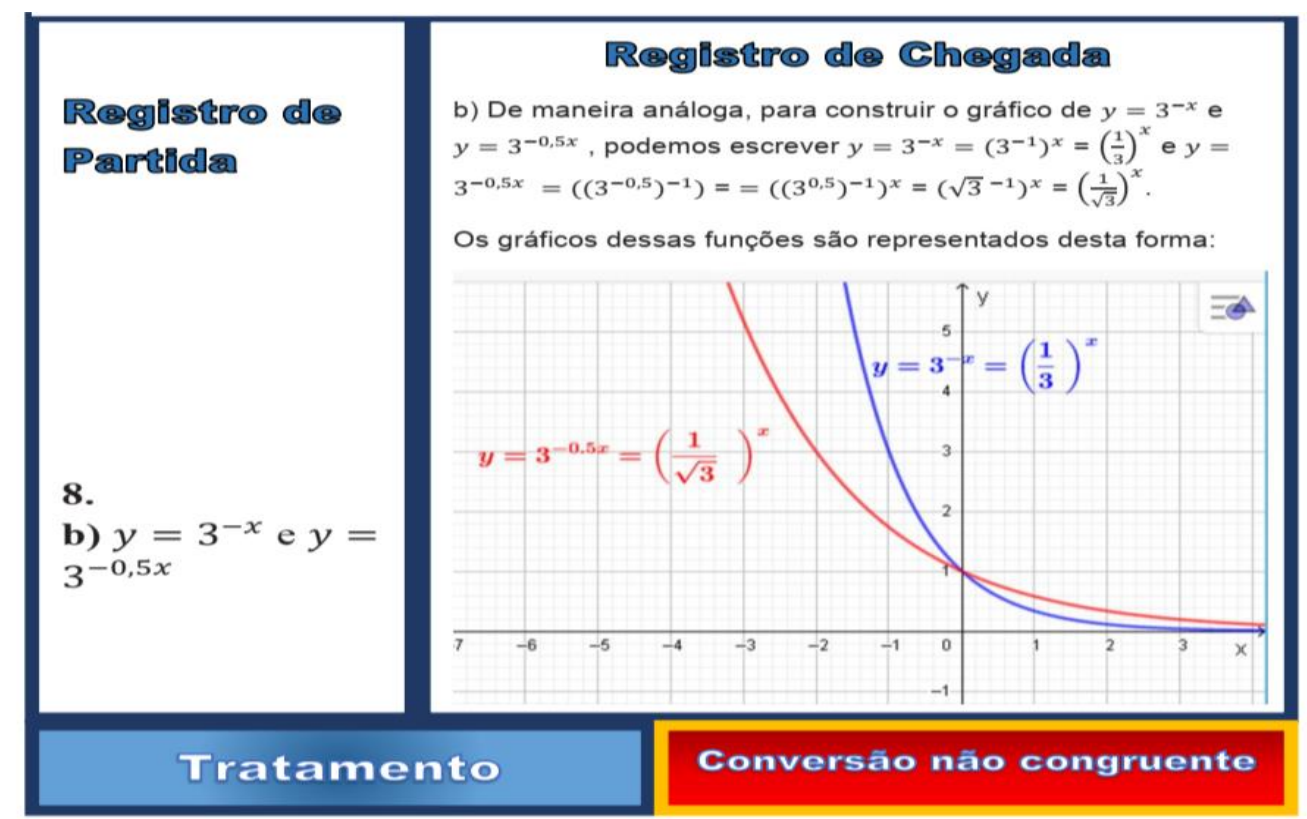

Fonte: Dados da pesquisa

Nesse sentido, constatamos que os termos significantes não se relacionam, não atendendo aos critérios de congruência de Duval (2009), constatando-se uma conversão não congruente, quando comparados as expressões algébricas e o resultado após o tratamento.

A atividade 8, itens c e d, analisada no estudo de Ginez (2020), assemelha-se às outras propostas de atividades. Pede-se a construção do gráfico e, na possível solução, realiza-se um tratamento a partir da transformação do registro de representação algébrico para o numérico, porém o resultado não transparece o valor inicial, ocorrendo também uma conversão não congruente.

As construções dos gráficos, com exceção do item a, não sugerem a construção de tabelas, apenas é pedido que se construa o gráfico, sem mencionar a forma e os recursos que podem ser utilizados.

Uma sugestão a ser trabalhada com os alunos nesses exercícios seria a utilização de recursos como o software para explorar os vários parâmetros apresentados pelas variadas expressões algébricas e suas construções gráficas. Todavia, não torna menos importante trabalhar as construções 
usando-se tabelas. Lembramos que as explorações dos diferentes registros tornam possíveis a compreensão dos alunos e a aquisição de conhecimento.

Na atividade 9, no Quadro 11, a expressão foi dada no enunciado e a proposta dos itens era a substituição de valores para encontrar o crescimento de uma população durante determinado tempo.

Quadro 11: Atividade 9 - Caderno do Professor

\begin{tabular}{|c|c|}
\hline 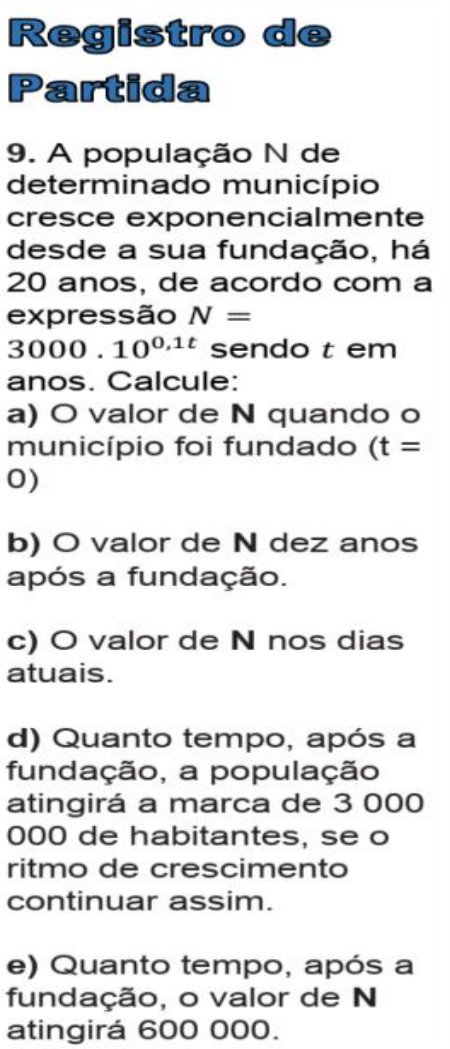 & 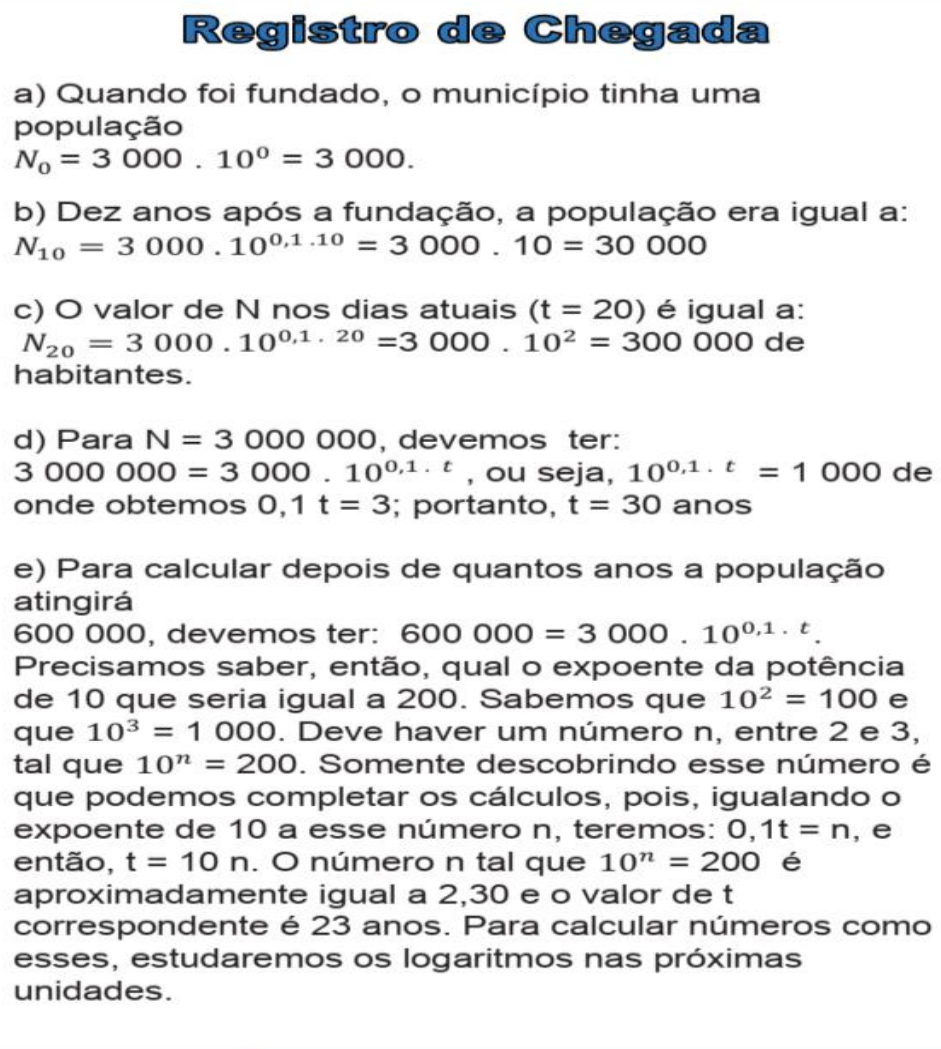 \\
\hline & Tratamento \\
\hline
\end{tabular}

Fonte: Dados da pesquisa

A princípio, o aluno necessita realizar uma interpretação do que foi proposto, identificando a relação com a expressão algébrica representada. Posteriormente, nos itens a, b e c, deve-se realizar a substituição dos valores dados em cada item na expressão algébrica e fazer os cálculos, isto é, uma transformação interna, considerada tratamento. Por sua vez, nos itens d e e, também ocorre um tratamento, porém, ao realizar os cálculos para determinar o valor de $10^{n}=200$, necessita-se de uma busca de valores aproximados.

A atividade apresenta um contexto que se aproxima do cotidiano dos alunos, o que pode favorecer o entendimento e a compreensão. Contudo, reforçamos que a assimilação de propriedades e, muitas vezes, a maneira de transitar entre os diferentes registros de representação facilitam o entendimento. 
Os registros apresentados em língua materna precisam ser explorados, pois muitas vezes os alunos não compreendem uma atividade em virtude da má interpretação feita do enunciado. Pensando no ensino de função exponencial, sentimos a necessidade de trabalhar com mais intensidade as propriedades de potência, para que não seja um empecilho no momento de os alunos transitarem entre os diferentes registros, além das abordagens que exigem dos alunos conhecimentos gráficos e algébricos para realizar uma interpretação global, que também são poucos exploradas.

\section{Considerações finais}

Neste estudo, optamos pela análise bibliográfica dos materiais, como o Caderno do Professor, por apresentarem situações que acabam levando o aluno a ter dificuldades em suas resoluções.

A análise do material permitiu evidenciar que as atividades propostas no caderno revelaram os diferentes registros de representação e que o fenômeno de congruência é encontrado em algumas abordagens.

No que tange às conversões não congruentes, acreditamos que elas necessitam ser trabalhadas com mais frequência, visto que são essenciais para o desenvolvimento de habilidades e competências adquiridas com atividades que abordam esse fenômeno. Salientamos a importância de estudar as propriedades das potências que colaboram para a aprendizagem e a aquisição do conhecimento para os diferentes registros de representação.

O estudo possibilitou identificar atividades que têm mais de uma transformação no registro de representação. Consideramos interessantes abordagens que explorem os registros de representação de forma gradativa, levando o aluno a compreender a transformação de tratamento; depois, uma conversão congruente; e, por fim, as conversões não congruentes, em que encontra uma maior dificuldade. Portanto, a nosso ver, uma das causas da não afinidade dos alunos com a Matemática são, muitas vezes, as abordagens que levam à incompreensão.

Por fim, ao refletir a respeito dos resultados e a partir da teoria de Duval, a qual salienta que a compreensão e os conhecimentos dos alunos serão adquiridos quando for possível transitar pelos diferentes registros, compreendeu-se a necessidade de elaborar e realizar atividades que privilegiem as conversões nos dois sentidos e que os variados registros necessitam ser explorados, principalmente com enfoques nas conversões congruentes e, em especial, nas não congruentes, que são as causas da incompreensão dos alunos e pouco exploradas. 


\section{Referências}

BOSCHESI, Fabio Henrique Lepri. Práticas pedagógicas com uso das TIC declaradas por professores de Matemática do Ensino Médio no contexto do novo currículo do Estado de São Paulo. 2016. 159 f. Dissertação (Mestrado) - Universidade Estadual Paulista, Presidente Prudente, 2016.

BRASIL, Secretaria de Educação Média e Tecnologia. PCN+ Ensino Médio: Orientações educacionais complementares aos Parâmetros Curriculares Nacionais: Matemática. Brasília: MEC/SEMTEC, <http://portal.mec.gov.br/seb/arquivos/pdf/CienciasNatureza.pdf> Acesso em: 02 jun. 2018.

BRASIL. Base Nacional Comum Curricular: Ensino Médio. Brasília: MEC/Secretaria de Educação Básica, $2018 . \quad$ Disponível em: http://portal.mec.gov.br/index.php?option=com_docman\&view=download\&alias=85121-bnccensino-medio\&category_slug=abril-2018-pdf\&Itemid=30192. Acesso em: 10 ago. 2019.

CASSIARI, Edna Ribeiro. Potencialidades e fragilidades na implementação do "Caderno do Professor" e "Caderno do aluno" da Rede Estadual de São Paulo. 2011. 104 f. Dissertação (Mestrado) - Pontifícia Universidade Católica de São Paulo, São Paulo, 2011.

DUVAL, Raymond. Semiósis e pensamento humano: registro semiótico e aprendizagens intelectuais (Sémiosis et Pensée Humaine: Registres Sémiotiques et Apprentissages Intellectuels). Tradução Lênio Fernandes Levy e Marisa Rosâni Abreu da Silveira. São Paulo: Editora Livraria da Física, 2009. Fascículo I.

DUVAL, Raymond. Gráficos e equações: a articulação de dois registros. Tradução Méricles Thadeu Moretti. Revista Eletrônica de Educação Matemática (REVEMAT), Florianópolis, v. 6, n. 2, p. 96-112, 2011a.

DUVAL, Raymond. Ver e ensinar matemática de outra forma: entrar no modo matemático de pensar os registros de representação semiótica. Organização Tânia M. M. Campos. Tradução Marlene Alves Dias. São Paulo: Proem, 2011b.

DUVAL, Raymond. Registros de representação semiótica e funcionamento cognitivo do pensamento. Trad. MORETTI, Méricles Thadeu. Revista Eletrônica de Educação Matemática (REVEMAT), Florianópolis, v. 7, n. 2, p. 266-297, 2012.

GIL, Antonio Carlos. Como elaborar um projeto de pesquisa. 4. ed. São Paulo: Atlas, 2002.

GINEZ, Patrícia Costa. Fenômeno de congruência e não congruência sobre a função exponencial em materiais didáticos. 2020. 107 f. Dissertação (Mestrado em Ensino de Ciências Exatas) Universidade Federal de São Carlos, Sorocaba, 2020.

RAMPINI, Elisabete Aparecida. Currículo e identidades docentes: o caso da proposta curricular da secretaria da educação do estado de São Paulo. 2011. 118 f. Dissertação (Mestrado) - Universidade Estadual de Campinas, Campinas, 2011.

SÃO PAULO. Secretaria da Educação. Material de apoio ao Currículo do Estado de São Paulo Caderno do Professor: 1. ${ }^{a}$ série do Ensino Médio, Matemática. São Paulo: SEE, 2014-2017. v. 2. 